\title{
An Empirical Evaluation of Security Indicators in Mobile Web Browsers
}

\author{
Chaitrali Amrutkar, Student Member, IEEE, Patrick Traynor, Member, IEEE, \\ and Paul C. van Oorschot, Member, IEEE
}

\begin{abstract}
Mobile browsers are increasingly being relied upon to perform security sensitive operations. Like their desktop counterparts, these applications can enable SSL/TLS to provide strong security guarantees for communications over the web. However, the drastic reduction in screen size and the accompanying reorganization of screen real-estate significantly changes the use and consistency of the security indicators and certificate information that alert users of site identity and the presence of strong cryptographic algorithms. In this paper, we perform the first measurement of the state of critical security indicators in mobile browsers. We evaluate ten mobile and two tablet browsers, representing over $90 \%$ of the market share, against the recommended guidelines for web user interface to convey security set forth by the World Wide Web Consortium (W3C). While desktop browsers follow the majority of guidelines, our analysis shows that mobile browsers fall significantly short. We also observe notable inconsistencies across mobile browsers when such mechanisms actually are implemented. We show where and how these failures on mobile browsers eliminate clues previously designed for, and still present in, desktop browsers to detect attacks such as phishing and man-in-the-middle. Finally, we offer advice on where current standards are unclear or incomplete.
\end{abstract}

Index Terms-Measurement, mobile security, SSL indicators, web browsers.

\section{INTRODUCTION}

Mobile browsers provide a rich set of features that often rival their desktop counterparts. From support for Javascript and access to location information to the ability for third-party applications to render content through WebViews, browsers are beginning to serve as one of the critical enablers of modern mobile computing. Such functionality, in combination with the near universal implementation of strong cryptographic tools including SSL/TLS, allows users to become increasingly reliant upon mobile devices to enable sensitive personal, social and financial exchanges.

In spite of the availability of SSL/TLS, mobile users are regularly becoming the target of malicious behavior. A 2011 report indicates that mobile users are three times more likely to access phishing websites than desktop users [19]. Security indicators (i.e., certificate information, lock icons, cipher selection, etc.) in web browsers offer one of the few defenses against such attacks. A user can view different security indicators and related certificate information presented by the browser to offer signals or clues about the credibility of a website. Although mobile and tablet browsers appear to support similar security indicators when compared to desktop browsers, the reasons behind the increasing number of attacks on mobile browsersare not immediately clear.

In this paper, we perform the first comprehensive empirical evaluation of security indicators in mobile web browsers. The

- This paper expands upon an earlier conference version [17]

- Chaitrali Amrutkar and Patrick Traynor are with the Georgia Tech Information Security Center (GTISC), Georgia Institute of Technology, Atlanta, GA 30332,USA.E-mail: chaitrali@,traynor@cc.gatech.edu

- Paul C. van Oorschot is with the School of Computer Science, Carleton University, 5302 Herzberg Building, 1125 Colonel By Drive, Ottawa, ON KIS 5B6, Canada. E-mail: paulv@scs.carleton.ca goal of this work is not to determine if average users take advantage of such cues, but instead whether security indicators are applied in a manner that allows expert users to accurately determine the identity of a website or verify the use of strong cryptographic primitives for communications. We believe that this distinction is critical because it highlights areas where not even the best trained users will be able to differentiate between malicious and benign behavior. Rather than an ad hoc analysis, we base our study on the recommendations set forward by the W3C for user interface security [10] as a proxy for best practices. In particular, we measure which browsers strictly conform to the absolute requirements ("MUST" clauses) and prohibitions ("MUST NOT" clauses). We perform our analysis across ten mobile and two tablet browsers, representing greater than $90 \%$ of the mobile market share [15], and then compare our results against the five most popular desktop browsers. Our experiments demonstrate that while the majority of desktop browsers largely meet the $\mathrm{W} 3 \mathrm{C}$ recommendations, all mobile browsers fail to meet many of the guidelines. Additionally, we observe that mobile browsers exhibit tremendous inconsistency in the presentation and availability of such indicators in contrast to traditional desktop browsers.

Our main contribution is a comprehensive and systematic evaluation and comparison of security indicators and security information for mobile and tablet browsers, to our knowledge the first such analysis undertaken. The main findings of our experiments are that all popular mobile and tablet browsers fail to meet, in numerous instances, the recommendations in the W3C guidelines for user interface of security information, whereas in comparison desktop browsers largely follow the guidelines. Our findings of tremendous inconsistency of user interfaces across mobile browsers, and between mobile and desktop browsers, are also expected to be of considerable interest. Among other contributions, we outline attacks on 
mobile browsers, such as phishing and undetectable manin-the-middle, enabled by failure to properly follow these guidelines; and we highlight missing security indicators, e.g., extended validation (EV) SSL indicators [9], [29], [33]. These are intended to convey an augmented assurance process, however we find most mobile browsers fail to implement EVSSL indicators visible to users, and their absence along with that of any distinguishing browser behavior, precludes EV-SSL certificates from providing relying parties any benefits beyond non-EV SSL certificates.

The remainder of our paper is organized as follows: Section 2 provides definitions and explains the mandatory elements of the W3C guidelines; Section 3 provides the primary results of our evaluation; Section 4 discusses secondary observations; Section 5 presents ways in that a user can be mislead about the identity of a website or the use of encryption and attacks that are enabled by this confusion; Section 6 presents an overview of related research; and Section 7 offers a discussion of our findings and concluding remarks.

\section{BACKGROUND: W3C RECOMMENDATIONS}

The World Wide Web Consortium (W3C) has defined user interface guidelines [10] for the presentation and communication of web security context information to end-users of both desktop and mobile browsers. For context in later sections, we first define the terminology and then provide a brief explanation of the $\mathrm{W} 3 \mathrm{C}$ guidelines referenced within this paper.

\subsection{Definitions}

User interface elements: User interface elements in browsers are divided in two categories [10]:

- Primary User Interface: the portions of a user interface that are available to users without being solicited by a user interaction. The primary user interface elements related to security traditionally include the padlock icon, the address bar, the https URL prefix, the favicon, and the site-identity button or URL coloring to signify the presence of EV-SSL and SSL certificates [9].

- Secondary User Interface: the portions of a user interface that are available to the user after they are solicited by a specific user interaction. The secondary user interface elements related to security include the security properties dialog, domain name, owner information, verifier information, information on why a certificate is trusted, validity period of manually accepted certificates (self-signed) and cipher details of an SSL connection.

Trust anchor: A trust anchor represents an authoritative entity represented by a public key and associated data. The public key is used to verify digital signatures and the associated data is used to constrain the types of information for which the trust anchor is authoritative. Relying parties (web browsers) use trust anchors to determine if digitally signed information objects are valid by verifying digital signatures using the trust anchor's public key and by enforcing the constraints expressed in the associated certificate data. Our interpretation is that a trust anchor refers to a certificate authority (CA).

Root: A root is a trust anchor that is any certificate authority (CA).

Trusted root: A trusted root is a CA whose public key is a priori trusted by the browser and may certify other keys.

Certificates: Public key certificates are widely used to provide keying material and convey a website's identity information to the user. The W3C defines four types of certificates. We provide our interpretation for the definitions of certificate types in the $\mathrm{W} 3 \mathrm{C}$ document where they are ambiguous. For additional information regarding the commercial practice of issuing and managing SSL certificates, please refer to the requirements defined by the CA/Browser forum [12].

- Validated certificate: This is a public key certificate that has been verified by chaining up to a trusted root. Our interpretation is that a standard SSL certificate signed by a CA trusted by a browser refers to a validated certificate.

- Augmented assurance certificate: The certificate chain for such a certificate MUST be validated up to a trusted root that is recognized as augmented assurance qualified by the user agent (user's browser). We interpret an EV-SSL certificate as an augmented assurance certificate that is validated by the browser.

- Self-signed certificate and untrusted root certificate: A self-signed certificate is a certificate that is signed by its own creator and is not a priori trusted by a browser. Our interpretation of an untrusted root certificate is that it refers to a certificate holding the public key of a CA, that is signed by a CA not a priori trusted by the user's browser.

- Interactively accepted trust anchors or certificates: This refers to either a CA or a website's public key that is accepted by a user and thereby used as a trust anchor by the browser. Whether the trust anchor is accepted just for the present transaction or for the present and the future transactions depends on the options presented to the user by the browser and then the option chosen by the user. When a browser receives a website certificate, the public key therein (and the certificate) is untrusted unless either the certificate was previously interactively accepted (for future sessions), or trust can be derived in it transitively, through a trust chain starting from a trust anchor (i.e., a CA key already trusted by the browser).

Pinning: Pinning associates one or more certificates with a specific website. The certificate provided by the website can either be self-signed or one issued by an untrusted root. Once a user interactively accepts such a certificate for the first time, the browser pins the certificate to the website. After pinning, the browser warns users only when the same website presents a different certificate. No warning messages are shown by the browser if a site shows a certificate consistent with previously pinned certificates for that site.

Identity Signal: An identity signal on a TLS-secured webpage includes information about the owner of the webpage and the certificate issuer's organization. A webpage's certificate 
provides its owner information and the issuer's (e.g., Certificate Authority) organization.

Strong TLS: An http transaction is strongly TLS-protected if it is TLS-protected, an https URL was used, strong TLS algorithms were negotiated for both confidentiality and integrity protection, and at least one of the following conditions is true: the server used a validated certificate that matches the dereferenced URI; the server used a self-signed certificate that was pinned to the destination; the server used a certificate chain leading to an untrusted root certificate that was pinned to the destination.

A strong TLS algorithm implies that no version of the TLS protocol that suffers known security flaws has been negotiated. Therefore, versions of SSL prior to SSLv3 MUST NOT be considered strong. Additionally, a strong TLS algorithm must also select a cipher suite for which key and algorithm strengths correspond to industry practice. More information on strong and weak TLS algorithms can be found in the W3C document [10] and RFC 4346 [4].

Weak TLS: An http transaction is weakly TLSprotected if it is TLS-protected, but strong TLS protection could not be achieved for one of the following reasons: TLS handshake used an anonymous key exchange algorithm, such as DH_anon; the cryptographic algorithms negotiated are not considered strong, such as TLS_KRB5_EXPORT_WITH_DES_CBC_40_SHA; certificates were used that are neither validated certificates nor self-signed certificates pinned to the destination.

Error messages: The W3C document defines common error interaction requirements and practices to signal two classes of errors ordered by increasing severity: warning/caution messages and danger messages.

Warning/caution messages are intended for situations when the system has reason to believe that the user may be at risk based on the current security context information, however a determination cannot positively be made. Danger Messages are intended for situations when there is a positively identified danger to the user (i.e., not merely a risk).

\subsection{W3C Guidelines}

We chose a subset of the absolute requirements (MUST) and prohibitions (MUST NOT) specified in the W3C user interface guidelines. ${ }^{1}$ We omitted the guidelines represented by clauses including the MAY, MAY NOT, SHOULD and SHOULD NOT keywords as they represent the optional guidelines [3]. We classify the $\mathrm{W} 3 \mathrm{C}$ guidelines into five categories: identity signal, certificates, TLS indicators, robustness and error messages.

\section{1) Identity signal: availability:}

The security indicators showing identity of a website MUST be available to the user either through the primary or the secondary interface at all times.

\section{2) Certificates: required content:}

1. The guidelines deemed to be the most critical and definitively testable were selected based on the authors' experience and knowledge of the area of SSL indicators.
In addition to the identity signal, the web browsers MUST make the following security context information available through information sources (certificates): the webpage's domain name and the reason why the displayed information is trusted (or not).

\section{3) TLS indicators:}

a) Significance of presence: Any UI indicator (such as the padlock) MUST NOT signal the presence of a certificate unless all parts of the webpage are loaded from servers presenting at least validated certificates over strongly TLSprotected interactions.

b) Content and Indicator Proximity: Content MUST NOT be displayed in a manner that confuses hosted content and browser chrome indicators, by allowing that content to mimic chrome indicators in a position close to them.

c) Availability: The TLS indicators MUST be available to the user through the primary or the secondary interface at all times.

\section{4) Robustness: visibility of indicators:}

Web content MUST NOT obscure the security user interface.

\section{5) Error messages:}

a) Interruption: Both warning/caution and danger messages MUST interrupt the user's current task, such that the user has to acknowledge the message.

b) Proceeding options: Warning/caution messages MUST provide the user with distinct options for how to proceed (i.e., these messages MUST NOT lead to a situation in which the only option presented to the user is to dismiss the warning and continue).

c) Inhibit interaction: The interactions for danger messages MUST be presented in a way that makes it impossible for the user to go to or interact with the destination website that caused the danger situation to occur, without first explicitly interacting with the danger message.

\section{Empirical Observations}

We evaluate ten mobile and two tablet browsers against the $\mathrm{W} 3 \mathrm{C}$ recommended practices for security indicators. The details of the browsers are provided in Table 1. For each of the guidelines described in Section 2.2, we create and run a set of experiments to verify compliance on all the candidate browsers and record our observations. All the experiments were performed on web browsers on real mobile phones, and are recreated in the respective emulators to generate many of the figures throughout the paper. The browser versions used in our evaluation are approximately the latest as of November $3^{r d}$, 2011. Table 2 through Table 6 provide the synopsis of the results of our experiments.

\subsection{Identity Signal: Availability}

An identity signal contains information about the owner of a website and the corresponding certificate issuer. Before issuing a certificate, the certificate provider requests the contact email 


\begin{tabular}{|c|c|c|c|c|c|}
\hline Category & Browser Name & Version & Rendering Engine & Operating System & Device \\
\hline \multirow{11}{*}{ Mobile } & Android & 2.3 .3 & $\begin{array}{c}\text { Webkit } \\
\end{array}$ & Android 2.3.3 & Nexus One \\
\hline & Blackberry & 5.0 .0 & Mango & Blackberry OS 5.0.0.732 & Bold 9650 \\
\hline & Blackberry & 6.0 .0 & Webkit & Blackberry OS 6 & Torch 9800 \\
\hline & Chrome Beta & 0.16 .4130 .199 & Webkit & Android 4.0.3 & Nexus S \\
\hline & Firefox Mobile & 4 Beta 3 & Gecko & Android 2.3 .3 & Nexus One \\
\hline & $\begin{array}{c}\text { Internet Explorer } \\
\text { Mobile }\end{array}$ & $*$ & Trident & $\begin{array}{l}\text { Windows Phone } \\
\text { 7.0.7004.0 OS }\end{array}$ & LG-C900 \\
\hline & Nokia Browser & 7.4.2.6 & Webkit & Symbian Belle & Nokia 701 \\
\hline & \multirow{2}{*}{ Opera Mini } & 6.0 .24556 & Presto & Android 2.3.3 & Nexus One \\
\hline & & 5.0 .019802 & Presto & iOS 4.1 (8B117) & iPhone \\
\hline & Opera Mobile & 11.00 & Presto & Android 2.3.3 & Nexus One \\
\hline & Safari & $*$ & Webkit & iOS 4.1 (8B117) & iPhone \\
\hline \multirow{2}{*}{ Tablet } & Android & $*$ & Webkit & Android 3.1 & Samsung Galaxy \\
\hline & Safari & $*$ & Webkit & iOS 4.3 .5 (8L1) & iPad 2 \\
\hline \multirow{5}{*}{ Desktop } & Chrome & 15.0 .874 .106 & Webkit & OS X 10.6.8 & - \\
\hline & Firefox & 7.0 .1 & Gecko & OS X 10.6.8 & - \\
\hline & Internet Explorer & 8.0 .7600 .16385 & Trident & Windows 7 & - \\
\hline & Opera & 11.52 & Presto & OS X 10.6.8 & - \\
\hline & Safari & 5.1 .1 & Webkit & OS X 10.6.8 & - \\
\hline
\end{tabular}

TABLE 1: Details of the browsers used for experimental evaluation. (*: The version numbers of these browsers were not apparent. We have used the default browsers shipped with the referenced version of the OS.)

address for the website from a public domain name registrar, and checks that published address against the email address supplied in the certificate request. Therefore, the owner of a website is someone in contact with the person who registered the domain name. Popular browsers represent the owner information of a website using different terminology including owner, subject, holder and organization.

We visited a public webpage presenting a trusted root certificate from all the candidate browsers. We then evaluated the browsers for the presence of identity signal, either on the primary or the secondary interface.

Observations: The IE Mobile, iPhone and iPad Safari, and Opera Mini and Mobile browsers do not provide a user interface to view certificates. Accordingly, the identity signal information is not available for a user of these browsers and thus none of these five browsers comply with the W3C guideline for availability of identity signal. We note that when a website presents a certificate that is from a CA not from a trusted root, all the browsers provide an interface to view the certificate via an error message. The Android mobile and tablet, Blackberry Mango and Webkit, Chrome Beta and Nokia browsers always allow a user to view certificates (both trusted and untrusted) and therefore comply with this guideline. A user is required to click the lock icon to view certificate information on the Chrome Beta and Blackberry Mango browsers. However, the browsers do not provide any visual indication to the user about this process of accessing the certificate information. Browsers supporting a UI for viewing certificate information provide a clear indication in the "options" in the browser menu. Although the Firefox Mobile browser does not support a certificate UI, it displays the identity information of a website when the site identity button is clicked, as shown in Figure 1. All desktop browsers comply with this guideline. Table 2 provides the summary of our results.

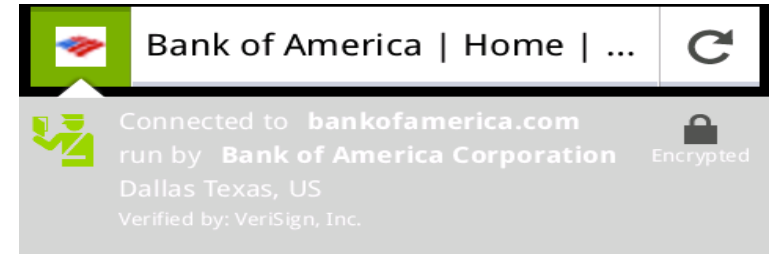

Fig. 1: Identity information displayed by Firefox Mobile.

\subsection{Certificates: Required Content}

In addition to the identity signal content, a certificate from a website must provide the same website's domain name and the reason why the displayed information is trusted (or not). Trust reasons include whether or not a certificate was accepted interactively, whether a self-signed certificate was used, whether the self-signed certificate was pinned to the site that the user interacts with, and whether trust relevant settings of the user agent were otherwise overridden through user action. We believe that information such as "certificate is implicitly trusted" and "the certificate chain is trusted/valid" also conveys the reason behind a browser trusting or not trusting a particular website.

We analyzed the candidate browsers for the presence of the required certificate content by visiting a website that uses strongly TLS-protected connection with its clients.

Observations: The IE Mobile, iPhone and iPad Safari, and Opera Mini and Mobile browsers do not provide a user interface to view certificates from trusted CAs. Therefore, these browsers fail to meet the $\mathrm{W} 3 \mathrm{C}$ guideline. Additionally, even though the remaining mobile and tablet browsers provide a user interface to view certificate information, they do not provide an explanation on why a particular certificate is trusted. Only the Blackberry Mango and Webkit browsers comply with the guideline by making all the required parts of a certificate available. When a website presents a certificate from a trusted CA, the Blackberry Mango and Webkit browsers 


\begin{tabular}{|c|c|c|c|c|}
\hline \multirow{2}{*}{$\begin{array}{c}\text { Mobile and Tablet } \\
\text { Browsers } \\
\text { (See Table } 1 \text { for versions) }\end{array}$} & \multicolumn{2}{|c|}{ 1) Identity signal: availability } & \multicolumn{2}{|c|}{ 2) Certificates: required content } \\
\hline & $\begin{array}{c}\text { Owner information } \\
\text { available? }\end{array}$ & $\begin{array}{c}\text { Certificate issuer's } \\
\text { information available? }\end{array}$ & $\begin{array}{c}\text { Domain name } \\
\text { available? }\end{array}$ & $\begin{array}{c}\text { Information on why certificate } \\
\text { trusted available? }\end{array}$ \\
\hline Android & $\bullet$ & $\bullet$ & $\bullet$ & $\times$ \\
\hline Blackberry Mango & $\bullet$ & $\bullet$ & $\bullet$ & $\bullet$ \\
\hline Blackberry Webkit & $\bullet$ & $\bullet$ & $\bullet$ & $\bullet$ \\
\hline Chrome Beta & $\bullet$ & $\bullet$ & $\bullet$ & $\times$ \\
\hline Firefox Mobile & $\bullet$ & $\bullet$ & $\bullet$ & $x$ \\
\hline iPhone Safari & $x$ & $\times$ & $x$ & $x$ \\
\hline Nokia Browser & $\bullet$ & $\bullet$ & $\bullet$ & $\times$ \\
\hline Opera Mini & $x$ & $\times$ & $x$ & $x$ \\
\hline Opera Mobile & $x$ & $x$ & $x$ & $x$ \\
\hline Windows IE Mobile & $x$ & $\times$ & $x$ & $x$ \\
\hline Safari on iPad 2 & $x$ & $\times$ & $x$ & $x$ \\
\hline Android on Galaxy & $\bullet$ & $\bullet$ & $\bullet$ & $x$ \\
\hline
\end{tabular}

TABLE 2: Results of experiments on candidate mobile browsers to test compliance with the first two W3C guidelines given in Section 2.2. Each guideline column consists of sub-columns stating the experiments performed on the browsers. An $\times$ implies that the browser does not comply with the respective $\mathrm{W} 3 \mathrm{C}$ guideline. A - implies that the browser complies with the respective W3C guideline. Note that all the desktop browsers are compliant to the same guidelines.

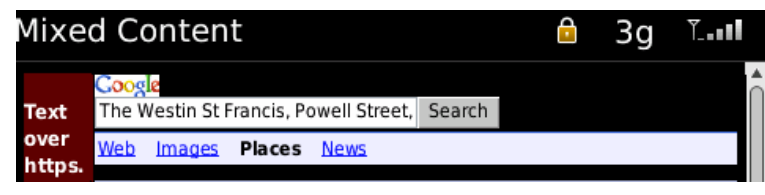

Fig. 2: Blackberry Mango browser rendering a mixed content webpage. Note that the webpage contains a Google map obtained over an http connection. Although the webpage holds mixed content, the browser displays the padlock icon as well as the https URL prefix indicators. This behavior fails to meet with guideline $3 \mathrm{a}$.

show the reason "certificate is implicitly trusted". Therefore, all but two mobile and tablet browsers fail to meet this W3C guideline. All desktop browsers follow this guideline correctly. Tables 2 and ?? provide the summary of our results.

\subsection{TLS Indicators}

TLS indicators include the https prefix, the padlock icon, information about the ciphers used in the connection and url coloring (or site identity button) to depict the difference between EV-SSL and SSL certified webpages.

a) Significance of presence: If a web browser displays a TLS indicator for the presence of a certificate for a webpage consisting of content obtained over both http and https connections (mixed content), this guideline is not followed.

We created a simple webpage that uses a strong TLS connection to retrieve the top level resource and embedded a map obtained from a third-party over an unsecured http connection. We analyzed the browsers while rendering the this page for two basic TLS security indicators: the https URL prefix and the padlock icon. If a browser shows any of these two indicators on a mixed content webpage, it does not follow the $\mathrm{W} 3 \mathrm{C}$ guideline. We also observed whether a browser shows a warning to the user suggesting the presence of mixed content on the webpage.

Observations: The Blackberry Mango, Blackberry Webkit and IE Mobile browsers display a lock icon on a webpage holding mixed content, thus failing to meet the $\mathrm{W} 3 \mathrm{C}$ guideline. Figure 2 shows a screen shot of the Blackberry Mango browser when a mixed content webpage is rendered. The Blackberry Webkit and IE Mobile browsers display a mixed-content warning and, if the user proceeds to the webpage, a lock icon is displayed. The Android browsers on the mobile and tablet devices present an open lock with a question mark inside the lock. The Chrome Beta browser displays a closed lock with a cross on top and a striked through https URL prefix for a mixed content webpage. This behavior of Android and Chrome is inconsistent with the other browsers. Therefore, it is necessary for the users of these browsers to understand the meaning of the new symbols in order to interpret its reference to mixed content on a webpage.

All the browsers display the https URL prefix either on the primary or the secondary interface. We note that this issue is present even in popular desktop browsers. The behavior of displaying the https URL prefix on a mixed content webpage fails to meet the $\mathrm{W} 3 \mathrm{C}$ recommendation in both the desktop and mobile environments as shown in Tables 3 and 4.

b) Content and Indicator Proximity: The padlock icon used as a security indicator and the favicon used as an identity element of a website are two popular elements that use a browser's chrome. If a browser allows a favicon to be placed next to the padlock, an attacker can feign a secure website by mimicking the favicon as a security indicator. We evaluate this scenario by visiting a webpage over a strong TLS connection from all candidate browsers and observed the relative locations of the favicon and padlock.

Observations: The Android mobile browser does not follow the $\mathrm{W} 3 \mathrm{C}$ guideline. The browser places the favicon of a webpage beside the padlock icon as shown in Figure 4. All other browsers adhere to this guideline, as shown in Tables 3 and 4.

We observed several inconsistencies in the use and position of the padlock icon and the favicon in the mobile and tablet 


\begin{tabular}{|c|c|c|c|c|c|c|}
\hline \multirow{3}{*}{$\begin{array}{c}\text { Mobile and Tablet } \\
\text { Browsers } \\
\text { (See Table } 1 \text { for versions) }\end{array}$} & \multicolumn{6}{|c|}{ TLS indicators } \\
\hline & \multicolumn{2}{|c|}{ 3a) significance of presence } & \multirow{2}{*}{$\begin{array}{l}\text { 3b) position } \\
\text { Favicon not next } \\
\text { to lock icon? }\end{array}$} & \multicolumn{3}{|c|}{ 3c) availability } \\
\hline & $\begin{array}{l}\text { Mixed content: } \\
\text { no lock shown? }\end{array}$ & $\begin{array}{c}\text { Mixed content: } \\
\text { no https shown? }\end{array}$ & & $\begin{array}{l}\text { https prefix } \\
\text { available? }\end{array}$ & Lock shown? & $\begin{array}{l}\text { Cipher details } \\
\text { available? }\end{array}$ \\
\hline Android & $\begin{array}{l}\text { Open lock with } \\
\text { a question mark }\end{array}$ & $x$ & $x$ & $\cdot(s)$ & $\bullet$ & $x$ \\
\hline Blackberry Mango & $x$ & $x$ & - & $\cdot(s)$ & - & - \\
\hline Blackberry Webkit & $x$ & $x$ & $\bullet$ & $\cdot(s)$ & $\bullet$ & $\bullet$ \\
\hline Chrome Beta & $\begin{array}{l}\text { Closed lock with } \\
\text { a cross on top }\end{array}$ & $\begin{array}{l}\text { https striked } \\
\text { through }\end{array}$ & - & $\cdot(s)$ & $\bullet$ & $\bullet$ \\
\hline Firefox Mobile & $\begin{array}{c}\text { No security } \\
\text { indicators shown }\end{array}$ & $x$ & - & - $(s)$ & $\begin{array}{l}\text { - On clicking the } \\
\text { site identity button }\end{array}$ & $x$ \\
\hline iPhone Safari & $\bullet$ & $x$ & $\bullet$ & $\cdot(s)$ & $\bullet$ & $x$ \\
\hline Nokia Browser & $\bullet$ & $x$ & $\bullet$ & $\cdot(s)$ & $\bullet$ & $x$ \\
\hline Opera Mini & $\bullet$ & $x$ & $\bullet$ & $\cdot(s)$ & $\bullet$ & $x$ \\
\hline Opera Mobile & $\bullet$ & $x$ & $\bullet$ & $\cdot(s)$ & $\bullet$ & $x$ \\
\hline Windows IE Mobile & $x$ & $x$ & $\bullet$ & $\cdot(s)$ & $\bullet$ & $x$ \\
\hline Safari on iPad 2 & $\bullet$ & $x$ & $\bullet$ & $\cdot(s)$ & $\bullet$ & $x$ \\
\hline Android on Galaxy & $\begin{array}{l}\text { Open lock with } \\
\text { a question mark }\end{array}$ & $x$ & $\bullet$ & $\bullet(s)$ & $\bullet$ & $x$ \\
\hline
\end{tabular}

TABLE 3: Results of experiments on candidate mobile browsers to test compliance with the W3C guidelines 3a, 3b, and 3c given in Section 2.2. The symbol notation is as defined in Table 2. 's': Implies that the https URL prefix is present on the 's'econdary interface.

\begin{tabular}{|c|c|c|c|c|c|c|}
\hline \multirow{3}{*}{$\begin{array}{l}\text { Desktop Browsers } \\
\text { (See Table } 1 \\
\text { for versions) }\end{array}$} & \multicolumn{6}{|c|}{ TLS indicators } \\
\hline & \multicolumn{2}{|c|}{ 3a) significance of presence } & \multirow{2}{*}{$\begin{array}{c}\text { 3b) position } \\
\text { Favicon not next } \\
\text { to lock icon? }\end{array}$} & \multicolumn{3}{|c|}{ 3c) availability } \\
\hline & $\begin{array}{l}\text { Mixed content: } \\
\text { no lock shown? }\end{array}$ & $\begin{array}{c}\text { Mixed content: } \\
\text { no https shown? }\end{array}$ & & $\begin{array}{c}\text { https prefix } \\
\text { available? }\end{array}$ & Lock shown? & $\begin{array}{c}\text { Cipher details } \\
\text { available? }\end{array}$ \\
\hline Chrome & $\begin{array}{l}\text { Lock with a } \\
\text { yellow triangle }\end{array}$ & $x$ & $\bullet$ & $\cdot(p)$ & - & $\bullet$ \\
\hline Firefox & $\bullet$ & $x$ & $\bullet$ & $\cdot(p)$ & $\begin{array}{l}\text { - On clicking the } \\
\text { site identity button }\end{array}$ & - \\
\hline IE & - & $x$ & - & $\bullet(p)$ & $\bullet$ & $x$ \\
\hline Opera & $\bullet$ & $x$ & $\bullet$ & $\cdot(p)$ & $\bullet$ & $\bullet$ \\
\hline Safari & $\bullet$ & $x$ & - & $\cdot(p)$ & $\bullet$ & $x$ \\
\hline
\end{tabular}

TABLE 4: Results of experiments on traditional web browsers to test compliance with the same guidelines as Table 3 . The symbol notation is as defined in Table 2. 'p': Implies that the https URL prefix is present on the 'p'rimary interface.

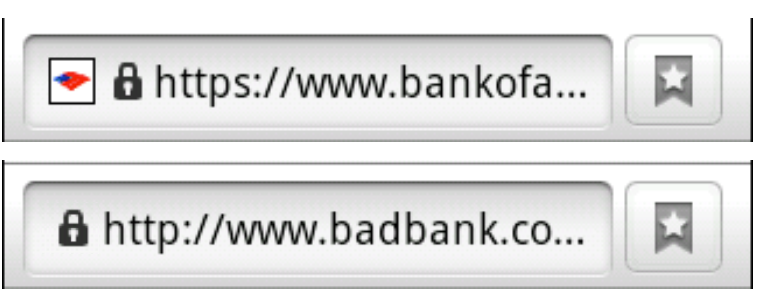

Fig. 4: The address bar of the Android browser when a webpage over SSL is loaded. The browser places the favicon adjacent to the lock icon, thereby violating the $\mathrm{W} 3 \mathrm{C}$ guideline $3 \mathrm{~b}$ described in Section 2.2. The star icon to the right of the address bar is to bookmark the webpage.

browsers. As shown in Figure 3, the favicon is displayed only on the Android (mobile and tablet), Blackberry Webkit and Firefox Mobile browsers. The remaining mobile and tablet browsers never display a favicon. This behavior is inconsistent with desktop browsers. We believe lack of screen space to be one of the drivers behind the removal of the favicon from the mobile environment. In addition to the almost total lack of use of favicons, we also noticed that the position of the padlock icon in mobile browsers is inconsistent across different mobile browsers. In the past, researchers have shown that the padlock icon is the security indicator most often noticed by users [24], [37]. Traditional desktop browsers generally display the padlock icon in the address bar. However, all mobile and tablet browsers except Android (mobile and tablet), Blackberry Webkit, Chrome Beta, and IE Mobile browsers display the lock icon on the title bar instead of the address bar. We believe that the reason behind this shift of location of the padlock icon in the mobile and tablet browsers is the nonpersistent availability of the address bar to the user. Whenever a user starts interacting with a webpage, most mobile browsers hide the address bar to accommodate more content on the small screen.

c) Availability: We studied the presence of the lock icon, the https URL prefix and details of the cipher used in a TLS connection by visiting a TLS protected webpage using all candidate browsers. The padlock icon and the https URL prefix are primary interface indicators and cipher information is a secondary interface indicator on desktop browsers. 

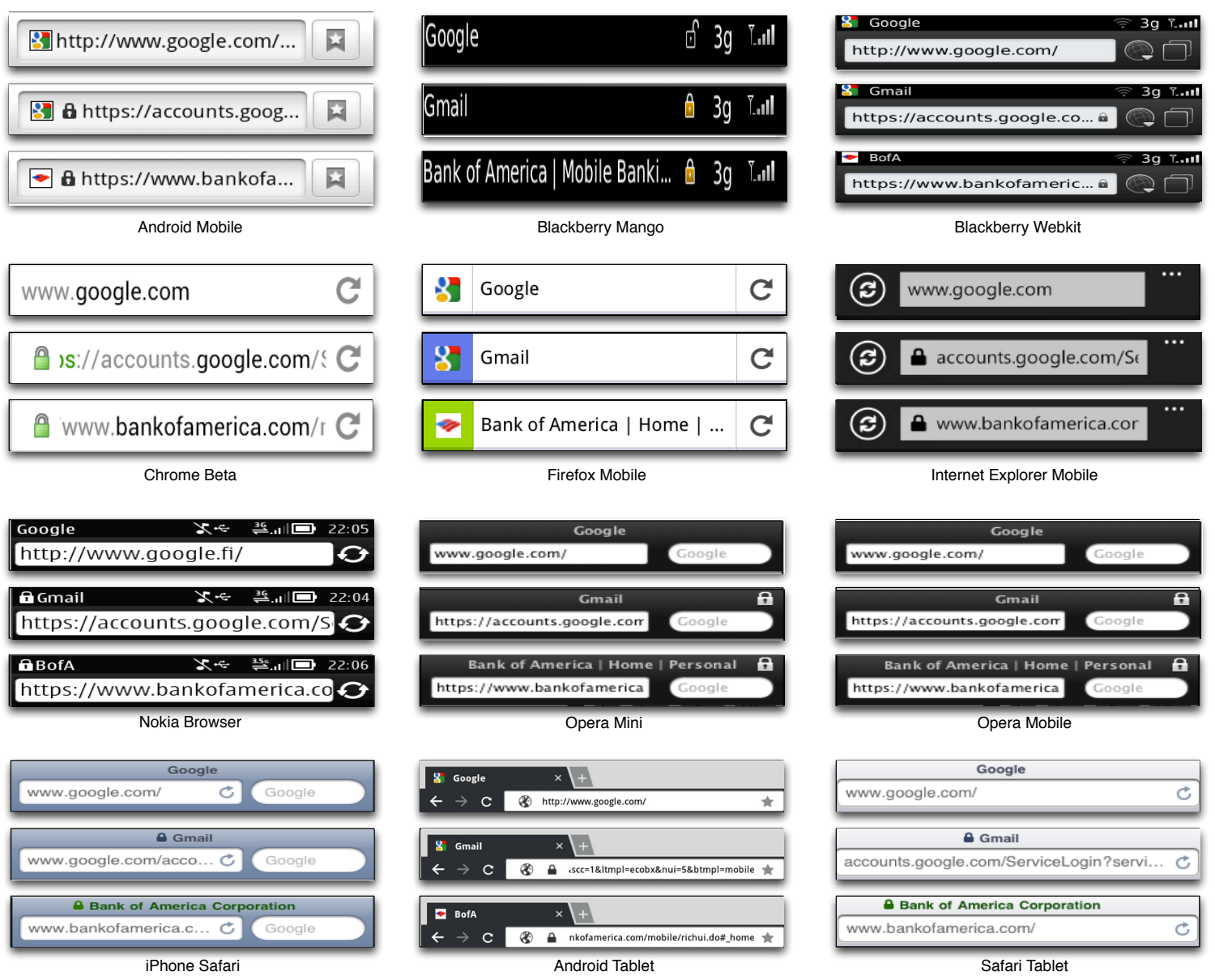

Fig. 3: Security indicators on the primary interface (address bar) of all the mobile and tablet browsers. Every browser has three screenshots of the address bar: from top to bottom, the websites are Google over an http connection, Gmail over a secure connection with an SSL certificate and Bank of America over a secure connection with an EV-SSL certificate.

Observations: Websites handling sensitive digital transactions (such as banks) ask users to search for the https URL prefix to ensure security of their transactions. Therefore, easy access to the https URL prefix is important. This indicator is present in the address bar (primary interface) of desktop browsers and is clearly visible to the user at all times. Among the mobile and tablet browsers, all but the Blackberry Mango browser display the https URL prefix in the address bar. The Blackberry Mango browser does not have an address bar and provides a choice to view the webpage's URL from the browser's options. This setting requires a user to be knowledgable of the change to be able to find the URL of the current webpage and also makes the https URL prefix a secondary interface indicator. Although the other mobile browsers display the https URL prefix in the address bar, they hide the address bar (except Chrome Beta) for better usability. In the Chrome Beta browser, if the URL of a webpage is longer than the screen size, the https URL prefix is hidden. Since a user is required to interact with the address bar to view the URL prefix of a webpage, the https URL prefix becomes a secondary interface indicator in all mobile and tablet browsers. This increases the likelihood of a successful downgrade attack (e.g., SSLstrip [7] attack) on the mobile and tablet browsers, since a user requires effort to view the https
URL prefix.

The information about the ciphers used in setting up the TLS connection between a website server and the user's browser is not available in any of the browsers except Blackberry Mango and Webkit. Accordingly, all the mobile and tablet browsers except two do not comply this $\mathrm{W} 3 \mathrm{C}$ guideline for our experiments. Tables 3 and 4 provide the summary of our results.

\subsection{Robustness: Visibility of Indicators}

The TLS indicators generally found on the primary interface are lock icon, https URL prefix, URL coloring and site identity button. Typically, the address bar in a web browser holds these indicators. Therefore, we examined whether web content overwrites or pushes the address bar containing security indicators out of a user's view during browsing.

Observations: Presumably, in order to free up screen real estate for other purposes, the address bar on all but one mobile browser is overwritten by web content once a webpage is rendered and/or when a user starts interacting with the page. The IE Mobile browser always displays the address bar, when the user accesses content in the portrait view. 


\begin{tabular}{|c|c|c|c|c|c|}
\hline \multirow{3}{*}{$\begin{array}{c}\text { Mobile and Tablet } \\
\text { Browsers } \\
\text { (See Table } 1 \text { for versions) }\end{array}$} & \multirow{3}{*}{$\begin{array}{l}\text { 4) Robustness } \\
\text { Content obscures } \\
\text { indicators on } \\
\text { the address bar? }\end{array}$} & \multicolumn{4}{|c|}{ 5) Error messages } \\
\hline & & \multicolumn{2}{|c|}{ 5a) Interruption } & \multirow{2}{*}{$\begin{array}{l}\text { 5b) Proceeding options } \\
\text { (for warnings) }\end{array}$} & \multirow{2}{*}{$\begin{array}{l}\text { 5c) Inhibit interaction } \\
\text { (for danger } \\
\text { messages) }\end{array}$} \\
\hline & & $\begin{array}{c}\text { Warning } \\
\text { (mixed content) }\end{array}$ & $\begin{array}{c}\text { Danger } \\
\text { (self-signed cert) }\end{array}$ & & \\
\hline Android & $x$ & $x^{*}$ & $\bullet$ & $N A^{*}$ & $\bullet$ \\
\hline Blackberry Mango & NA & $x^{*}$ & $\bullet$ & $N A^{*}$ & $\bullet$ \\
\hline Blackberry Webkit & $\times$ & $\cdot$ & $\bullet$ & $\begin{array}{l}\text { "Continue, Close connection, } \\
\text { View cert, Trust cert" options }\end{array}$ & $\bullet$ \\
\hline Chrome Beta & $x$ & $x^{*}$ & $\bullet$ & $N A^{*}$ & $\bullet$ \\
\hline Firefox Mobile & $x$ & $x^{*}$ & $\cdot$ & $N A^{*}$ & $\bullet$ \\
\hline iPhone Safari & $\times$ & $x^{*}$ & $\bullet$ & $N A^{*}$ & $\bullet$ \\
\hline Nokia Browser & $x$ & $\cdot$ & $\bullet$ & $\bullet$ & $\bullet$ \\
\hline Opera Mini & $\times$ & $x^{*}$ & $\times$ & $N A^{*}$ & $\times$ \\
\hline Opera Mobile & $x$ & $x^{*}$ & $\bullet$ & $N A^{*}$ & $\bullet$ \\
\hline Windows IE Mobile & $x$ & $\cdot$ & $\bullet$ & $\begin{array}{l}\text { "Yes and No" } \\
\text { options }\end{array}$ & $\bullet$ \\
\hline Safari on iPad 2 & $\bullet$ & $x^{*}$ & $\bullet$ & $N A^{*}$ & $\bullet$ \\
\hline Android on Galaxy & $x$ & $\bullet$ & $\bullet$ & $\begin{array}{c}\text { "Continue, View Certificate, } \\
\text { Go Back" options }\end{array}$ & $\bullet$ \\
\hline
\end{tabular}

TABLE 5: Results of experiments on traditional web browsers to test compliance with the W3C guidelines 4, 5a, 5b and 5c given in Section 2.2. The symbol notation is as defined in Table 2. $N A$ : Implies that the concerned experiment is not applicable to that browser, the reasoning can be found in the text. (*: Our view is that a browser should display a warning message for a webpage holding mixed content, to avoid misleading users trained to interpret SSL indicators to mean that the (entire) webpage is secured.) $x^{*}$ : Implies that the browser fails to warn a user according to our view.

\begin{tabular}{|c|c|c|c|c|c|}
\hline \multirow{3}{*}{$\begin{array}{c}\text { Desktop Browsers } \\
\text { (See Table } 1 \text { for versions) }\end{array}$} & \multirow{3}{*}{$\begin{array}{l}\text { 4) Robustness } \\
\text { Content obscures } \\
\text { indicators on } \\
\text { the address bar? }\end{array}$} & \multicolumn{4}{|c|}{ 5) Error messages } \\
\hline & & \multicolumn{2}{|c|}{ 5a) Interruption } & \multirow{2}{*}{$\begin{array}{l}\text { 5b) Proceeding options } \\
\text { (for warnings) }\end{array}$} & \multirow{2}{*}{$\begin{array}{l}\text { 5c) Inhibit interaction } \\
\text { (for danger } \\
\text { messages) }\end{array}$} \\
\hline & & $\begin{array}{c}\text { Warning } \\
\text { (mixed content) }\end{array}$ & $\begin{array}{c}\text { Danger } \\
\text { (self-signed cert) }\end{array}$ & & \\
\hline Chrome & - & $\begin{array}{c}\times^{*} \\
\text { site identity button } \\
\text { shows a warning }\end{array}$ & - & $N A^{*}$ & - \\
\hline Firefox & - & $x^{*}$ & - & $N A^{*}$ & - \\
\hline IE & $\bullet$ & - & $\bullet$ & $\begin{array}{c}\text { "Yes, No" } \\
\text { More info options }\end{array}$ & $\bullet$ \\
\hline Opera & - & $x^{*}$ & - & $N A^{*}$ & - \\
\hline Safari & - & $x^{*}$ & - & $N A^{*}$ & - \\
\hline
\end{tabular}

TABLE 6: Results of experiments on traditional web browsers to test compliance with the same guidelines as Table 5. The symbol notation is as defined in Table 2 and Table 5.

However, the address bar is never displayed in IE Mobile when a user interacts with a webpage in the landscape mode. The Chrome beta browser makes the address bar persistently available in both the portrait and landscape modes. Out of the two tablet browsers, only the tablet Safari browser avoids the security indicators on the address bar being overwritten by a webpage's content, therefore allowing a persistent view of the security indicators on the primary interface. The Android tablet browser hides the address bar once a webpage is rendered. Tables 5 and 6 show that all the candidate desktop browsers follow this guideline unlike the mobile and tablet browsers.

\subsection{Error Messages}

We created example scenarios that demand the warning/caution and danger messages, given the definitions in the $\mathrm{W} 3 \mathrm{C}$ document. The $\mathrm{W} 3 \mathrm{C}$ document provides examples of scenarios that demand a danger alert. However, as the document does not specify any scenarios that should trigger warnings, we carried out our tests using the following scenario.
We classified the scenario of a browser rendering a mixed content webpage as one that should trigger a warning. This is because on a webpage with both insecure and secure content, the user may or may not interact with the insecure content on the webpage. Therefore, the browser system is unable to positively determine whether the user is at risk. In contrast, we used an example scenario given in the $\mathrm{W} 3 \mathrm{C}$ document for our experiments on danger messages. The $\mathrm{W} 3 \mathrm{C}$ document defines 'rendering a webpage presenting a self-signed certificate' as one that should trigger a danger message, since the certificate is not from a trusted root.

a) Interruption: We examined whether the mobile and tablet browsers display a warning or danger message in our test scenarios. We further observed the nature of the messages to confirm that they actually interrupt the user's actions as specified by the $\mathrm{W} 3 \mathrm{C}$ guidelines and are not displayed at a position on the screen which a user can ignore and continue interacting with the website. 
Observations: Only four mobile and tablet browsers (Android Galaxy, Blackberry Webkit, IE Mobile and Nokia) display a warning notifying the user of the existence of insecure content on a mixed content webpage, before the webpage is rendered. The other browsers do not interrupt the user by displaying a warning. The iPhone Safari browser shows a mixed content warning on a console that needs to be enabled by a user and is intended for developers. We believe that most iPhone Safari users are unlikely to enable the debug console, carefully browse the debug messages and therefore understand the presence of mixed content. Among the desktop browsers, only IE displays a mixed content warning, thereby interrupting a user.

The mobile and tablet browsers comply with the interruption guideline by displaying a danger message, when a webpage with a self-signed certificate is rendered. The Opera Mini browser is the only browser that does not display a danger message in this scenario; it simply renders the webpage and does not show any TLS indicators.

b) Proceeding options: We examined whether the warning message displayed for a mixed content webpage provides a user with more than one option to proceed after interruption.

Observations: Only the Android Galaxy, Blackberry Webkit, IE Mobile and Nokia browsers display a warning message when navigated to a mixed content webpage. The IE Mobile browser informs the user about the presence of unsecured content on the webpage and provides two options for continuing: <Yes, No> . However, there is no option to the user to view the certificate provided by the top-level website using a secured connection. Conversely, the Android Galaxy and Nokia browsers provide an option to view a website's certificate. The options presented by the Android Galaxy browser are $<$ Continue, View Certificate, Go back $>$ and those presented by the Nokia browser are $<$ Options, Back $>$. The "Go back" and "Back" options navigate the user to a webpage viewed right before the mixed content webpage. The options provided by the Nokia browser are <Accept this time only, Accept permanently, Certificate details $>$. The Blackberry Webkit browser provides the options to $<$ Continue, Close Connection (default), View Certificate, Trust Certificate $>$. Among the desktop browsers, IE provides $<$ Yes, No, More info $>$ options to proceed when a mixed content webpage is rendered.

c) Inhibit interaction: This guideline requires a browser to prevent a user from interacting with a website that triggers a danger message, before user interaction with the danger message. We visited a website presenting an untrusted self-signed certificate from all the browsers.

Observations: All mobile and tablet browsers except Opera Mini display a danger message on receiving a self-signed certificate. Additionally, they restrict a user's interaction to the danger message. A user is unable to access the website content before explicitly interacting with the danger message. Figure 5 shows the danger message presented by the iPhone

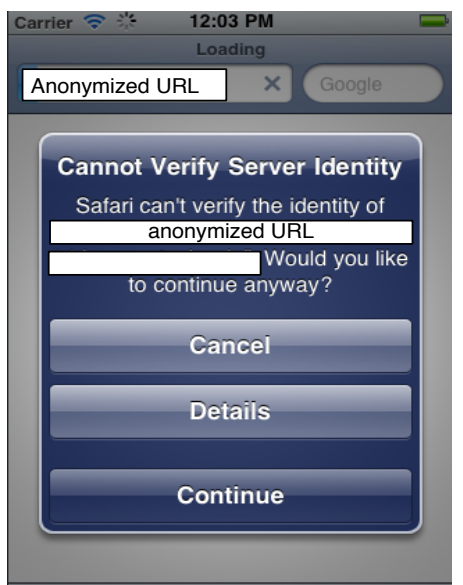

Fig. 5: Danger message on iPhone Safari when a website presenting a self-signed certificate is accessed. This message interrupts the user and also inhibits the user from proceeding without interacting with the danger message first. Note that the website's URL has been anonymized for submission.

Safari browser before loading a webpage with a self-signed certificate. The Opera Mini browser does not show an error for self-signed certificates. It simply routes the user to the webpage presenting the self-signed certificate. All desktop browsers correctly follow this guideline. Table 5 and Table 6 summarize our experimental results of the error message guidelines.

\section{Additional RESUlts}

We discuss our findings that are not directly related to the guidelines studied in Section 3. We note our observations on the positive and negative characteristics shown by the mobile and tablet browsers. We also discuss an important security scenario that is not represented in the $\mathrm{W} 3 \mathrm{C}$ guidelines and argue that it requires attention. Table 7 provides a summary of the results covered in this section.

\subsection{The Good}

The W3C document defines two guidelines that MUST hold when strong TLS algorithms are negotiated between a client and a server:

1) No version of the TLS protocol that suffers known security flaws has been negotiated. At the point of writing of this document, versions of SSL prior to SSLv3 MUST NOT be considered strong.

2) A cipher suite has been selected for which key and algorithm strengths correspond to industry practice. The "export" cipher suites explicitly prohibited in appendix A.5 of TLSv11 [4] (RFC 4346) MUST NOT be considered strong.

To verify the compliance with these guidelines we conducted two experiments.

SSLv2: We browsed to a website supporting only SSLv2 from each of the candidate browsers. We found that all the mobile, tablet and desktop browsers comply with the first guideline and do not support SSLv2. 


\begin{tabular}{|c||c|c|c|c|}
\hline $\begin{array}{c}\text { Mobile and Tablet } \\
\text { Browsers } \\
\text { (See Table 1 for versions) }\end{array}$ & $\begin{array}{c}\text { SSLv2 } \\
\text { supported? }\end{array}$ & $\begin{array}{c}\text { Null cipher } \\
\text { supported? }\end{array}$ & $\begin{array}{c}\text { Weak cipher } \\
\text { prohibited? } \\
\text { (DES-CBC-SHA) }\end{array}$ & $\begin{array}{c}\text { EV-SSL vs SSL } \\
\text { differentiation? }\end{array}$ \\
\hline \hline Android & $\bullet$ & $\bullet$ & $\times$ & $\times$ \\
\hline Blackberry Mango & $\bullet$ & $\bullet$ & $\times$ & $\times$ \\
\hline Blackberry Webkit & $\bullet$ & $\bullet$ & $\times$ & $\times$ \\
\hline Chrome Beta & $\bullet$ & $\bullet$ & $\bullet$ & $\times$ (site identity \\
\hline Firefox Mobile & $\bullet$ & $\bullet$ & $\bullet$ & $\begin{array}{c}\text { (title } \\
\text { URL coloring) }\end{array}$ \\
\hline iPhone Safari & $\bullet$ & $\bullet$ & $\times$ & $\times$ \\
\hline Nokia Browser & $\bullet$ & $\bullet$ & $\times$ & $\times$ \\
\hline Opera Mini & $\bullet$ & $\bullet$ & $\bullet$ & $\times$ \\
\hline Opera Mobile & $\bullet$ & $\bullet$ & $\bullet$ & $\times$ (title \\
\hline Windows IE Mobile & $\bullet$ & $\bullet$ & $\bullet$ & $\times$ \\
\hline Safari on iPad 2 & $\bullet$ & $\bullet$ & $\bullet$ & URL coloring) \\
\hline Android on Galaxy & $\bullet$ & $\bullet$ & & $\times$ \\
\hline
\end{tabular}

TABLE 7: Results of the support for SSLv2, the null cipher, DES-CBC-SHA (weak cipher) and whether browsers differentiate between EV-SSL and SSL certified webpages. The symbol notation is as defined in Table 2.

Null cipher: The null cipher is one of the prohibited ciphers in RFC 4346 and one of the most dangerous ciphers because it represents the lack of an encrypted communication channel. To test browser compliance with the second guideline for strong TLS algorithms, we built a website that supports only the null cipher. We observed that none of the mobile, tablet or desktop candidate browsers support the null cipher. ${ }^{2}$

Discontinuing support for SSLv2 and the null cipher automatically reduces the probability of cipher downgrade attacks on the candidate browsers.

\subsection{The Bad}

A browser supporting a weak cipher can enable a network attacker to break the encrypted messages offline. The SSLv3 cipher-suite consists of certain weak ciphers, although they are stronger than the SSLv2 ciphers and the null cipher. We verified the support of the DES-CBC-SHA weak cipher. We observed that six (Android Mobile, Blackberry Mango and Webkit, iPhone and iPad2 Safari and Nokia Browser) out of the eleven mobile and tablet browsers support the weak cipher. The other mobile and tablet browsers display error messages conveying the absence of a common encryption protocol with the server. It is interesting to note that the the Safari browser in its mobile, tablet and even desktop versions supports this weak cipher. However, the Android tablet browser does not support this cipher, unlike its mobile version. Since most mobile and tablet browsers do not allow users to see the cipher used on a TLS connection, they can not determine that a weak cipher is being used. No desktop browser other than Safari support this cipher.

\subsection{The Silent}

The $\mathrm{W} 3 \mathrm{C}$ document does not establish guidelines for the browser user interface to signify the difference between EV-

2. We did not test for the support to all the prohibited ciphers (as given in TLSv11 [4]) by the candidate browsers.
SSL [9], [33] and SSL certificates. The sole distinction between an SSL and an EV-SSL certificate from a user's perspective is the set of indicators on his browser. For example, the Firefox desktop browser uses a green site identity button to convey the presence of an EV-SSL certificate on a website. However, the site identity button is blue in the same browser when a website with an SSL certificate is rendered.

SSL certificates can be 'domain-validation-only' with minimal verification performed on the details of the certificate. Since any successful SSL connection causes the padlock icon to appear, users are not likely to be aware of whether the website owner has been validated or not. Therefore, fraudulent websites have started using inexpensive domain-validated SSL certificates with minimal verification to gain user trust. EV-SSL certificates were created to restore confidence among users that a particular website has been subjected to more rigorous vetting and has a verifiable identity. If browsers do not differentiate between SSL and EV-SSL certificates, then the fundamental motivation [9] behind EV-SSL certificates becomes void, so too does the incentive for site owners to pay extra for such certificates. An SSL certificate from Go Daddy costs \$12.99/year [1] and an EV-SSL certificate from VeriSign costs \$1499/year [2]. In a browser with no differentiation between SSL and EV-SSL, both these certificates are the same from a user's perspective. An adversary holding a domain name and willing to spend money for the SSL certificate would then trigger exactly the same user interface elements to users, and thus appear to provide identical guarantees as a website certified by the more expensive certificate.

Experimental observations: We browsed both EV-SSL and SSL certified webpages using all the candidate browsers. With the exceptions of the Firefox Mobile and the iPhone and iPad Safari browsers, none of the mobile or tablet browsers display any indicators that differentiate between EV-SSL and SSL certified webpages. The Firefox Mobile browser uses 
green and blue colors of the site identity button to depict the presence of EV-SSL and SSL certified webpages respectively. The Safari mobile and tablet browsers use green and blue coloring of the 'title' to represent the difference between EVSSL and SSL. This behavior of the Firefox Mobile and the Safari browsers is consistent with their desktop counterparts. However, the IE Mobile, and the Opera Mini and Mobile browsers are not consistent with the methods used on their desktop counterparts to portray the different between EV-SSL and SSL webpages.

Gauging the security level of a website using the different EV-SSL and SSL indicators can be complicated for an average user. The inconsistency across the mobile and desktop browsers from the same vendor adds to an already confusing task. We believe that a clear guideline on the indicators for differentiating between EV-SSL and SSL certified webpages is necessary to help browser vendors provide the expected interface consistently in the desktop and mobile environments. Moreover, we suggest that a guideline from a well established international standards organization such as the $\mathrm{W} 3 \mathrm{C}$ is a minimal starting point in order to achieve consistency across browser software from different vendors.

We note the following advice within official guidelines from the CA/Browser Forum [6]: In cases where the relying application accepts both EV and non-EV certificates, it is recommended that the application's behavior differ in a distinct way for each type of certificate. Application developers should consider the EV treatment offered by other application developers that also recognize EV certificates and, where practical, provide consistent treatment. We believe that much more specific advice is essential, for example, in a revision or extension of the W3C user interface guidelines [10].

\section{User Deception and Potential At- TACKS}

The $\mathrm{W} 3 \mathrm{C}$ user interface guidelines are an effort to communicate security information to users such that they can make informed decisions about websites that they visit. If these guidelines are not implemented by a browser, users are more easily misled about the identity of a website or the security of a connection. We first discuss five methods for user deception, followed by four attacks that an adversary can launch using one or more of the five deception methods. Table 8 provides a summary of potential attacks described in this section on the candidate browsers.

\subsection{Deception Methods}

We discuss techniques to confuse user perception about the security of a website's pages when rendered in a browser that fails to meet one or more of the $\mathrm{W} 3 \mathrm{C}$ guidelines. A malicious website or a network attacker successful in deceiving a user is more likely to succeed in stealing a user's sensitive information (i.e., phishing). We assume that a malicious website principal or a network attacker has knowledge of the incomplete security indicators in popular mobile browsers. We also assume that an honest user visits a website using a mobile browser that does not follow one or more of the $\mathrm{W} 3 \mathrm{C}$ guidelines discussed in
Section 2.2. Violation of a single W3C guideline may permit multiple attack vectors. The goal of an adversary is to trick the user into believing that they are interacting with a secure website when they are actually interacting with an insecure one.

1) Close imitation of identity information: $A$ malicious website can closely imitate the identity of a legitimate website to confuse a user. Attackers often buy domains that very closely resemble a legitimate website's domain in addition to imitating the content of the legitimate website. For example, an attacker can buy the domain "bankofamericaa.com" that closely resembles the "bankofamerica.com" domain (the malicious one has an additional 'a' at the end of bankofamerica). Difficulty in clearly viewing a website's URL due to the constrained screen size of a mobile device allows for the possibility of a user overlooking the slight difference in the domain name. Additionally, the attacker can also obtain an inexpensive SSL certificate for his malicious website so that the browser shows SSL indicators to the user to confuse the user into believing that the malicious website is the legitimate one.

An expert user can view a website's identity, domain name and reason behind trusting the website, presented in the website's certificate to identify the true owner of the phishing website and avoid divulging sensitive information. Absence of the identity information of a website, domain name and the reason of trusting that particular website certificate is a violation of guideline 1 and 2 given in Section 2.2.

2) Lock icon spoofing: The padlock icon is an important TLS indicator on the primary interface of a browser. The padlock combined with the presence of the https prefix in the URL signifies the presence or absence of SSL. The placement of the padlock icon is critical because it provides a clean indication of encryption and therefore security. If browsers situate a site's favicon next to the padlock icon in the primary interface, the utility of the padlock icon is diminished.

A malicious website can make its favicon appear exactly the same as a user's browser's padlock icon. This provides the illusion of strong TLS encryption and allows an attacker to convince an expert user that his personal information (e.g., credit card number) is kept confidential and encrypted in transit. Moreover, the fake padlock makes the website appear more legitimate without the attacker purchasing any SSL certificates. Finally, a browser allowing an attacker to manipulate the contents of the security indicators with website content is a direct violation of guideline $3 \mathrm{~b}$ described in Section 2.2.

3) Cipher downgrade: A man-in-the-middle (network attacker) can tamper with the initial messages sent by a client browser to establish an SSL connection with a website server and force the negotiation of a weak cipher. An expert user can refrain from providing sensitive information on a connection established using a weak cipher, by viewing the cipher negotiated for the TLS connection with the server. However, the same expert would be unable to detect the presence of a weak cipher on an SSL connection carrying sensitive data if the browser does not display cipher information of the connection. Such a browser fails to meet the $\mathrm{W} 3 \mathrm{C}$ requirement $3 \mathrm{c}$ in Section 2.2. 


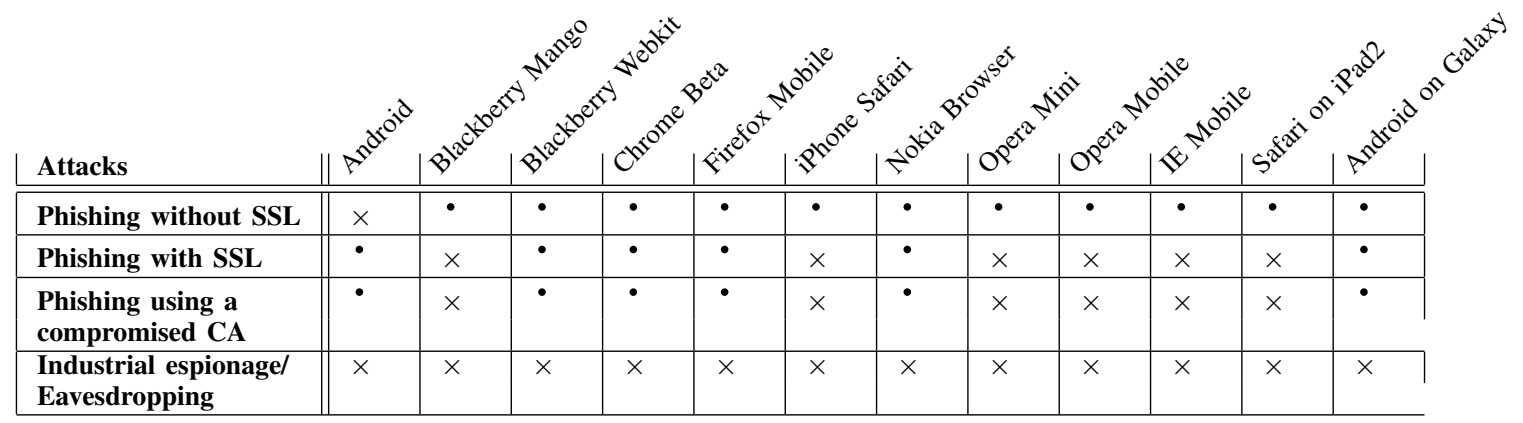

TABLE 8: Summary of potential attacks on candidate mobile browsers. A $\times$ implies that the attack is possible. A $\cdot$ implies that the corresponding attack is not possible on the browser.

4) Substitute http for https: The https URL prefix is a TLS security indicator signifying encryption on the channel between the client and the server. Since the https URL prefix is available on the primary user interface (address bar), a user can easily view this indicator while browsing sensitive data. If a network attacker changes the intended https website to an http website, a user may be able to recognize that he is using an unsecured connection by noticing the change from https to http in the address bar.

If the https prefix is not available to a user persistently, requirement 3c described in Section 2.2 is not followed.

5) Mixed content: The content of a webpage is interpreted as mixed if the top-level resource was retrieved through a strongly TLS protected http transaction and some dependent resources were retrieved through a weakly protected or unprotected http transaction. Dependent resources include inline images, stylesheets, script content, and frame content. If a browser displays security indicators defined for strongly TLS secured webpages on a webpage hosting mixed content, users gain a false notion of security for the unsecured content. This can lead to users providing sensitive information such as passwords while interacting with unsecured http content embedded in a webpage whose top resource is acquired over a strongly protected TLS connection. Moreover, this browser behavior does not comply with the $\mathrm{W} 3 \mathrm{C}$ guideline $3 \mathrm{a}$ given in Section 2.2.

\subsection{Attacks}

An attacker can exploit one or more of the above techniques for user deception to launch a range of attacks. We describe four attacks in order of increasing effort required from an attacker.

i) Phishing without SSL: An attacker masquerades as a trustworthy entity in a phishing attack. By closely imitating a legitimate website's identity information in combination with lock icon spoofing, a malicious website can launch a phishing attack without SSL as follows.

An attacker buys a domain name that closely resembles the domain name of the legitimate website. The attacker imitates the content of the targeted legitimate website. Instead of spending money on purchasing an SSL certificate to increase the "false" credibility of the malicious website, an attacker instead makes the favicon of the malicious website a lock image. Therefore, the closely imitated domain name provides an impression of correct identity of the intended website and the spoofed lock provides an illusion of strong encryption.

When this malicious website is rendered in a browser that makes viewing the URL of the website difficult and does not offer a UI to view identity information such as website owner's name, even an advanced user might be subjected to phishing. ii) Phishing with SSL: Spoofing only the lock icon may not be adequate to launch a successful phishing attack. To increase the credibility of a phishing website, the attacker can buy a inexpensive SSL certificate for the website. The presence of a valid certificate causes a browser to display SSL indicators such as the https URL prefix and URL coloring/colored site identity button in addition to the lock icon in the browser's chrome. If a user blindly trusts just these SSL indicators and can not verify additional identity information of the website, he can be subjected to a phishing attack.

iii) Phishing using a compromised CA: Compromising a CA allows an attacker to obtain rogue certificates for legitimate websites. There have been several such attacks recently [13], [14]. If a CA is trusted by a user's browser, all certificates signed by the CA will be accepted by the browser without showing any warning to the user. This behavior persists even when the same CA is compromised and the necessary update to remove the trusted CA from the browser has not been installed. An expert user who is knowledgable of a CA compromise can verify every certificate issuer's organization in the certificate chain, therefore declining interacting with a malicious website with a rogue certificate. If a browser does not present user interface to enable certificate viewing, even an expert user could be exposed to a phishing attack.

iv) Industrial espionage / eavesdropping: A man-in-themiddle (network) attacker can use any one of the cipher downgrade, substituting http for https or inserting mixed content techniques for user deception to launch an eavesdropping attack on a user's session as follows:

SSLstrip attack: The SSLstrip [7] man-in-the-middle attacker sits on a local network and intercepts traffic. When the attacker detects a request to an encrypted https site, he substitutes a duplicate of the intended destination as an unencrypted http site. This switching strips away the security that prevents a third party from stealing or modifying data, while deceiving the server that an encrypted page has been sent to the client. The network attacker can also fake a lock icon in the stripped http page, by replacing the favicon by a lock icon [30].

Cipher downgrade attack: A man-in-the-middle (network attacker) can tamper with the initial messages sent by a client browser to establish an SSL connection with a website server. Before a TLS connection is set up, a client and server exchange a list of ciphers that they support. A network attacker can 
modify the list of supported ciphers sent by the client to a list containing only weak ciphers, and then forward the client's request/response to the server. On receiving a list of only weak ciphers (e.g., DES-CBC-SHA), the server can either drop the connection because no ciphers are mutually supported, or provide support for that cipher and begin an encrypted session with the weak cipher. When a connection using the weak cipher is initiated, all the data in transit is protected using the weak cipher's encryption scheme. This allows a network attacker to capture the stream of data and break the weak encryption offline. The attack is also useful to mislead even an expert user that their transactions are over a connection with strong encryption algorithms, since the SSL indicators such as https URL prefix and lock icon are present even for a connection using a weak cipher.

Mixed content attack: A man-in-the-middle attacker can tamper (e.g., code injection) with the unencrypted content present on a webpage consisting of mixed content and replace the original content with any malicious content of his choice. If a web browser displays SSL indicators for a webpage containing mixed content, even an expert user would be unable to detect a network attack exploiting the mixed content on a webpage.

The results of the experiments discussed in Section 3 combined with the threat model defined in this section make the candidate mobile and tablet browsers susceptible to phishing and eavesdropping attacks as shown in Table 8.

\section{Related Work}

TRADITIONAL BROWSER INDICATORS: Traditional desktop browsers contain a range of security indicators in the chrome of the browser including the lock icon, the https URL prefix, and certificates. Several studies have indicated that these security cues used in desktop browsers go unnoticed [23], [24], [32], [33], [37] or are absent in websites [34]. Although domain name mismatches between certificates and websites are observed often [36], Sunshine et al [35] showed that users ignore TLS warnings for domain name mismatches, and showed that users ignore TLS warnings for expired certificates and unknown CAs. Moreover, a majority do not understand these warnings. The lock icon is the security indicator most often noticed [24], [37]. However, even when used as a security cue by users, many do not fully understand its meaning [22]-[24] and its absence also often goes unnoticed [23]. Additionally, the majority of users who rely on the lock icon remain unaware of its identity feature [23], [24], [26], [37] and do not reliably understand the concept of certificates [22], [23]. Indicators for newer technologies such as EV-SSL have also been shown to be ineffective to convey better security to the user as compared to a simple SSL certificate [18], [29].

TECHNIQUES FOR BETTER INDICATORS: Several techniques have been proposed to design better security indicators to prevent potential attacks such as phishing and web spoofing. Researchers have proposed better warnings [35], more effective interface dialogues [18], browser plugins [20], trusted path from the browser to the human user [38] and mandatory security indicators [27] to help users make correct security decisions. Other proposed security mechanisms include disabling JavaScript in the user browser and forcing persistent visibility of the browser's location line [25]. Dynamic Security Skins [22] allow a remote web server to prove its identity in a way that is easy for a human user to verify and hard for an attacker to spoof. Finally, efforts have been taken [6], [9], [10], [12], [16] to standardize security indicators and thus minimize confusion across browsers.

MOBILE BROWSER INDICATORS: Almost all the efforts in the area of security indicators in browsers have been focused on desktop browsers. The increasing user base of mobile web browsers and mobile e-commerce has made mobile browsers attractive targets for attacks [5], [8], [11], [21], [31]. In light of these developments and considering how the mobile browser user interface differs from desktops, it is important to analyze and understand the security indicators used in mobile browsers. Although the W3C [10] guidelines consider mobile browsers in their definitions, a large scale evaluation of the state-of-the-art security indicators in mobile browsers has not been carried out.

\section{Discussion and Concluding Remarks}

\subsection{Discussion}

For this study, we selected a subset of the absolute requirements and prohibitions from the W3C guidelines. From our experimental analysis, we observed that popular mobile and tablet browsers fail to meet many of the guidelines. However, by and large popular desktop browsers follow the set of guidelines studied in this paper. Furthermore, the inconsistencies that we observed herein are cause for significant concern, as consistency of user interfaces is recognized as a fundamental usability attribute [28], conveying many user benefits including enhancing users' ability to transfer skills across similar systems, and reducing training time on new and related systems. Moreover, related to security, inconsistency confuses users, and confusion aids the attacker. Our results raise an important question: is it appropriate to apply the same user interface guidelines for web security to both the desktop and the mobile environments?

We believe that the non-conformance to the $\mathrm{W} 3 \mathrm{C}$ guidelines and the inconsistencies in the use and presentation of SSL indicators in mobile browsers is primarily due to the adjustments made in the browser interface as a result of the tension between usability and security, and possibly due to dis-connects between mobile and desktop development teams. For example, the address bar consisting of the padlock icon and the https URL prefix indicators is persistently available in desktop browsers, however is hidden (apparently to better accommodate content on small mobile screens) for the majority of the time during user interaction. It is cumbersome for a user to bring the address bar in view (to observe indicators) by scrolling to the top of the mobile screen, suggesting this will be done far less frequently, whereas viewing the indicators on a desktop browser requires little or no extra effort. This ease of interaction with desktop browsers also makes consuming certificate information simpler for a user. Again in contrast, current design decisions related to mobile screen 
real estate force users to execute scrolling operations to view all the content of a certificate, implying greater inconvenience and effort compared to consuming certificate information on desktop browsers; in other cases, mobile browser vendors have decided to make certificate information entirely unavailable. Such significant design changes preclude even expert users from discerning clues about the credibility and security of websites, due to the absence of security indicators, leaving average users with no hope at all. These security concerns, the very significant non-conformance with existing recommendations, and tremendous inconsistency both within and across browser vendors, lead us to call for the establishment of new recommendations for the mobile environment that specifically take into account its limitations and additional challenges.

\subsection{Conclusion}

Modern mobile browsers enable a range of sensitive operations over SSL/TLS connections. Although these browsers aim for equivalent functionality to traditional desktops, their smaller screen size has resulted in significant changes to the presentation and availability of SSL indicators. We have carried out the first evaluation of security indicators in mobile browsers, using the $\mathrm{W} 3 \mathrm{C}$ web interface guidelines to measure compliance in ten mobile and two tablet browsers. We observed that mobile browsers fail to meet many of the security guidelines and exhibit tremendous inconsistency in the presentation and availability of SSL indicators in contrast to traditional desktop browsers. Such significant design changes preclude even expert users from discerning clues about the credibility and security of websites, raising significant concerns about the security of average users. Additionally, we observed that the absence of clear and consistent EV-SSL indications leads to EV-SSL certificates currently adding complexity to the mobile ecosystem without any corresponding benefits. Our work may be viewed as a call to arms for greater consistency in mobile browser security interfaces and greater attention to the specific challenges of mobile device issues in security user interface guidelines. It also raises questions about the utility and usability of security indicators as presently implemented in mobile browsers and related questions about the viability of extended validation SSL certificates in light of current mobile browser user interfaces.

\section{ACKNOWLEDGMENTS}

This work was supported in part by the US National Science Foundation (CNS-0916047, CAREER CNS-0952959). Any opinions, findings, conclusions or recommendations expressed in this publication are those of the authors and do not necessarily reflect the views of the National Science Foundation. The third author is supported in part by an NSERC Discovery Grant, and NSERC ISSNet.

\section{RefEREnCES}

[1] GoDaddy SSL certificate. http://www.godaddy.com/Compare/gdcompare_ssl. aspx ?isc $=$ sslqgo016b.

[2] VeriSign certificate. https://www.verisign.com/ssl/buy-ssl-certificates/index.html? sl=t72010166130000002 $\backslash \&$ gclid=CIKMyY2GuKgCFYg32godV2 8Bw

[3] Key words for use in RFCs to Indicate Requirement Levels. http://www.ietf.org/ rfc/rfc2119.txt, March 1997.
[4] The Transport Layer Security (TLS) Protocol Version 1.1. http://www.ietf.org/ rfc/rfc4346.txt, April 2006.

[5] Overflow clickjacking. http://research.zscaler.com/2008/11/ clickjacking-iphone-style.html, November 2008.

[6] Guidelines for the Processing of EV Certificates, version 1.0. http://www. cabforum.org/Guidelines_for_the_processing_of_EV_certificatesv1_0.pdf, January 2009.

[7] SSLstrip, presented at Black Hat DC. http://www.thoughtcrime.org/software/ sslstrip/, 2009.

[8] Android Browser Exploit. http://threatpost.com/en_us/blogs/ researcher-publishes-android-browser-exploit-110810, 2010.

[9] Guidelines For The Issuance And Management Of Extended Validation Certificates, version 1.3. http://www.cabforum.org/Guidelines_v1_3.pdf, November 20 2010.

[10] W3C: Web Security Context: User Interface Guidelines. http://www.w3.org/TR/ wsc-ui/, August 2010.

[11] Web-based Android attack. http://www.infoworld.com/d/security-central/ security-researcher-releases-web-based-android-attack-317?source=rss security_central/, November 2010.

[12] Baseline Requirements for the Issuance and Management of Publicly-Trusted Certificates, version 1.0. http://www.cabforum.org/Announcement-Baseline Requirements.pdf, April 112011.

[13] Comodo compromise. http://www.csoonline.com/article/678777/ comodo-compromise-expands-hacker-talks, April 12011.

[14] DigiNotar CA compromise. http://community.websense.com/blogs/securitylabs/ archive/2011/08/30/diginotar-ca-compromise.aspx, August 302011.

[15] Mobile Browser Market Share. http://gs.statcounter.com/\#mobile browser-ww-monthly-201011-201111, November 2011

[16] The CA/Browser forum. http://www.cabforum.org/, April 112011.

[17] C. Amrutkar, P. Traynor, and P. C. van Oorschot. Measuring SSL Indicators on Mobile Browsers: Extended Life, or End of the Road? In Proceedings of the Information Security Conference (ISC), 2012.

[18] R. Biddle, P. Van Oorschot, A. Patrick, J. Sobey, and T. Whalen. Browser interfaces and extended validation SSL certificates: an empirical study. In Proceedings of the ACM workshop on Cloud computing security, 2009.

[19] M. Boodaei. Mobile users three times more vulnerable to phishing attacks. http://www.trusteer.com/blog/ mobile-users-three-times-more-vulnerable-phishing-attacks, 2011.

[20] N. Chou, R. Ledesma, Y. Teraguchi, D. Boneh, and J. Mitchell. Client-side defense against web-based identity theft. In Proc. NDSS, 2004.

[21] C. Davies. iPhone Os Safari Vulnerable To DoS Attacks. http://www.iphonebuzz. com/iphone-safari-dos-bug-discovered-162212.php, April 162008

[22] R. Dhamija and J. Tygar. The battle against phishing: Dynamic security skins. In Proceedings of the symposium on Usable privacy and security, 2005.

[23] R. Dhamija, J. D. Tygar, and M. Hearst. Why phishing works. Proceedings of the SIGCHI conference on Human Factors in computing systems, 2006.

[24] J. Downs, M. Holbrook, and L. Cranor. Decision strategies and susceptibility to phishing. In Proceedings of the Second Symposium on Usable Privacy and Security, 2006.

[25] E. W. Felten, D. Balfanz, D. Dean, and D. S. Wallach. Intrusion Detection Prevention Web Spoofing: An Internet Con Game. In 20th National Information Systems Security Conference, 1997.

[26] B. Friedman, D. Hurley, D. Howe, E. Felten, and H. Nissenbaum. Users' conceptions of web security: a comparative study. In CHI extended abstracts on Human factors in computing systems, 2002.

[27] A. Herzberg and A. Jbara. Security and identification indicators for browsers against spoofing and phishing attacks. ACM Transactions on Internet Technology, 2008

[28] J. Nielsen. Usability Engineering. Morgan Kaufmann, 1993.

[29] C. Jackson, D. Simon, and D. Tan. An evaluation of extended validation and picture-in-picture phishing attacks. Financial Cryptography and Data, 2007.

[30] M. Marlinspike. More Tricks For Defeating SSL In Practice. http://www.blackhat.com/presentations/bh-usa-09/MARLINSPIKE/ BHUSA09-Marlinspike-DefeatSSL-SLIDES.pdf, 2009.

[31] J. Resig. iPhone overflow clickjacking. http://ejohn.org/blog/ clickjacking-iphone-attack/, November 2008.

[32] S. Schechter, R. Dhamija, A. Ozment, and I. Fischer. The Emperor's New Security Indicators. IEEE Symposium on Security and Privacy, 2007.

[33] J. Sobey, R. Biddle, P. van Oorschot, and A. Patrick. Exploring user reactions to new browser cues for extended validation certificates. Computer SecurityESORICS, 2008.

[34] D. Stebila. Reinforcing bad behaviour: the misuse of security indicators on popular websites. In Proceedings of the 22nd Conference of the Computer-Human Interaction Special Interest Group of Australia on Computer-Human Interaction, 2010.

[35] J. Sunshine, S. Egelman, H. Almuhimedi, N. Atri, and L. F. Cranor. 18th USENIX Security Symposium Crying Wolf: An Empirical Study of SSL Warning Effectiveness. Work, 2009.

[36] N. Vratonjic, J. Freudiger, V. Bindschaedler, and J.-P. Hubaux. The inconvenien truth about web certificates. In The Workshop on Economics of Information Security (WEIS), 2011.

[37] T. Whalen and K. Inkpen. Gathering evidence: use of visual security cues in web browsers. In Proceedings of Graphics Interface, 2005.

[38] Z. E. Ye, S. Smith, and D. Anthony. Trusted paths for browsers. ACM Transactions on Information and System Security (TISSEC), May 2005. 